\title{
Structure of $I_{3} R$ Channel: High-resolution Insights from Cryo- EM
}

\author{
Mariah R. Baker, Guizhen Fan, and Irina I. Serysheva \\ Structural Biology Imaging Center, Department of Biochemistry and Molecular Biology, McGovern \\ Medical School at The University of Texas Health Science Center at Houston, 6431 Fannin Street, \\ Houston, TX 77030, USA
}

\begin{abstract}
Inositol 1,4,5-trisphosphate receptors $\left(\mathrm{IP}_{3} \mathrm{Rs}\right)$ are ubiquitously expressed intracellular $\mathrm{Ca}^{2+}$ channels and the major mediators of cellular $\mathrm{Ca}^{2+}$ signals generated by the release of $\mathrm{Ca}^{2+}$ ions from intracellular stores in response to a variety of extracellular stimuli. Despite established physiological significance and proven involvements of $\mathrm{IP}_{3} \mathrm{R}$ channels in many human diseases, detailed structural basis for signal detection by these ion channels and their gating remain obscure. Recently, single particle electron cryomicroscopy (cryo-EM) has yielded a long-awaited nearatomic resolution structure of the entire full-length type $1 \mathrm{IP}_{3} \mathrm{R}$. This structure provided exciting mechanistic insights into the molecular assembly of $\mathrm{IP}_{3} \mathrm{R}$, revealing the pronounced structural conservation of $\mathrm{Ca}^{2+}$ release channels and raising many fundamental and controversial questions on their activation and gating. Here we summarize the major technological advances that propelled our cryo-EM analysis of $\mathrm{IP}_{3} \mathrm{R}$ to near-atomic resolution and discuss what the future holds for structural biology of $\mathrm{Ca}^{2+}$ release channels.
\end{abstract}

\section{Introduction}

The release of $\mathrm{Ca}^{2+}$ ions from intracellular stores, such as endoplasmic/sarcoplasmic (ER/SR) reticulum, is the most versatile cellular signaling mechanism that is responsible for numerous and markedly different cellular actions including gene transcription, secretion, muscle contraction, learning and memory, cell proliferation and cell death. Intracellular $\mathrm{Ca}^{2+}$ release is mediated by $\mathrm{Ca}^{2+}$ release channels, inositol 1,4, 5-trisphosphate receptors ( $\mathrm{IP}_{3} \mathrm{Rs}$ ) and ryanodine receptors (RyRs), which are enormous tetrameric membrane protein assemblies. Dysfunction of these ion channels has been linked to a wide range of neurodegenerative and neuromuscular diseases characterized by abnormal intracellular $\mathrm{Ca}^{2+}$ homeostasis [1,2]. Defining the molecular mechanisms for $\mathrm{Ca}^{2+}$ signals generated upon activation of $\mathrm{Ca}^{2+}$ release channels is therefore a major goal in the quest for understanding health and pathology in virtually all living cells. Accomplishment of this goal is dependent

Corresponding author: Irina Serysheva, McGovern Medical School at The University of Texas Health Science Center at Houston, MSB 6.210, Houston, TX 77030, USA; irina.i.serysheva@uth.tmc.edu; Phone: +1 (713) 500 - 5523; Fax: +1 (713) 500 - 6297.

Publisher's Disclaimer: This is a PDF file of an unedited manuscript that has been accepted for publication. As a service to our customers we are providing this early version of the manuscript. The manuscript will undergo copyediting, typesetting, and review of the resulting proof before it is published in its final citable form. Please note that during the production process errors may be discovered which could affect the content, and all legal disclaimers that apply to the journal pertain. 
upon our knowledge of the detailed protein structure, thus enabling mechanistic insights into channel function.

Understanding structural basis of ion transport through biological membranes is an important and challenging frontier in structural biology. Ion channels are integral membrane proteins that are embedded within the lipid bilayer membrane and responsible for molecular transport through the membranes. These proteins have been proven to be much more difficult targets for structural analysis than their soluble counterparts, mainly because of their instability outside biological membranes. To date, structural biology is taking advantage of recent technical advances in the cryo-EM field (reviewed in [3-5]), and single particle cryo-EM is increasingly becoming the most applied method for structure determination of dynamic multi-domain biological assemblies, which have not been amenable to the traditionally employed structural techniques of crystallography and NMR spectroscopy. In favorable cases, the achievable resolution for single particle cryo-EM is now comparable to that produced in atomic-resolution crystallographic studies, 2-3 $\AA$ [610]. Starting in 2013, single particle cryo-EM made remarkable strides in solving structures of a wide spectrum of integral membrane proteins at near-atomic (3-5 $\AA$ ) resolutions [1118 ] including the megadalton $\mathrm{Ca}^{2+}$ release channels, $\mathrm{IP}_{3} \mathrm{Rs}$ and RyRs [19-25].

However, structural analysis of IP3R poses unique challenges for single particle cryo-EM largely due to genuine heterogeneity of the channel complexes present in native tissues and their flexible multi-domain architecture. The $\mathrm{IP}_{3} \mathrm{R}$ family is comprised of three homologous isoforms (types 1-3) encoded by separate genes [26,27]. Although all $\mathrm{IP}_{3} \mathrm{R}$ isoforms exhibit strong sequence homology ( $70 \%$ sequence identity), especially within the TM regions ( $90 \%$ sequence identity), they harbor significant differences in the regulatory cytoplasmic domains. These differences likely underlie distinct isoform-specific channel properties in terms of channel modulation by endogenous and exogenous ligands and tissue distribution. $\mathrm{IP}_{3} \mathrm{Rs}$ are expressed in virtually all mammalian tissues and their diversity is further expanded by the homo- and hetero-tetrameric assembly of the $\mathrm{IP}_{3} \mathrm{R}$ protomers into functional channels, as well as by alternative gene splicing [26,27]. Moreover, the presence of glycosylation and phosphorylation sites at the cytosolic and lumenal faces of the channel protein may also produce conformational variations in vivo. As such, preparations of completely structurally homogeneous $\mathrm{IP}_{3} \mathrm{R}$ samples from native sources is not achievable given that most mammalian cell types express more than one $\mathrm{IP}_{3} \mathrm{R}$ isoform, and expression of such large recombinant protein suitable for high-resolution structural analysis remains challenging. Advantageous to structural studies are cerebellar Purkinje cells that express predominantly the type $1 \mathrm{IP}_{3} \mathrm{R}$ isoform, which is the most functionally and structurally characterized member of the $\mathrm{IP}_{3} \mathrm{R}$ family.

In this review, we summarize the technical and methodological advances that have enabled near-atomic resolution structure determination of $\mathrm{IP}_{3} \mathrm{R} 1$ by single-particle cryo-EM [19]. We also discuss the latest structural insights into the $\mathrm{IP}_{3} \mathrm{R}$ architecture, which uncover mechanistic details of $\mathrm{Ca}^{2+}$ release channels, and consider future prospects and challenges for structural studies of these channels. 


\section{Brief Retrospective on the $I_{3} R$ structural studies}

During the past decade, structural information on the $\mathrm{IP}_{3} \mathrm{R}$ protein has been obtained by three major structural techniques: X-ray crystallography [28-31], NMR spectroscopy [32,33] and single-particle cryo-EM [19,34-40]. However, given the exceptionally large size of the entire $\mathrm{IP}_{3} \mathrm{R}$ channel $(\sim 1.2 \mathrm{MDa})$, only single particle cryo-EM was able to tackle the structure of the full-length tetrameric channel assembly. Over a decade ago, several lowresolution structures of the entire $\mathrm{IP}_{3} \mathrm{R} 1$ were reported almost simultaneously [34-38] yet, none agreed, even with respect to the overall shape of the channel, leading to uncertainty over the true 3D structure of $\mathrm{IP}_{3} \mathrm{R} 1$. Further cryo-EM studies allowed our group to unambiguously establish the $3 \mathrm{D}$ architecture of the entire $\mathrm{IP}_{3} \mathrm{R} 1$ channel and clearly prove the veracity of the new structure to intermediate resolution (10-15 $\AA$, depending on local flexibility in specific domains) by applying multiple validation methods [39,40], including tilt validation [41,42], class-average/map comparisons, independent refinements in multiple software packages and 'gold standard' resolution evaluation method [43]. Critical methodological improvements in protein purification and cryospecimen preparation protocols greatly aided the progress of structural studies of $\mathrm{IP}_{3} \mathrm{R} 1$ and resulted in images with better contrast than those previously achieved. The root of discrepancies between the earlier conflicting EM studies of $\mathrm{IP}_{3} \mathrm{R} 1$ [34-38] was confirmed to arise from insufficient contrast in the raw data underlying the $3 \mathrm{D}$ reconstructions [40,44]. These key studies [39,40] laid to rest a critical controversy on the $\mathrm{IP}_{3} \mathrm{R} 1$ quaternary structure, however the lack of a high-resolution $3 \mathrm{D}$ structure of the entire $\mathrm{IP}_{3} \mathrm{R} 1$ significantly limited our ability to understand the structure-function relationship underlying ligand-induced channel activation and to rationalize its interactions with multiple modulatory molecules in a cellular context. Most recently, significant technical advances in the cryo-EM field have enabled structure determination of $\mathrm{IP}_{3} \mathrm{R} 1$ at near-atomic resolution allowing further critical insights into the gating machinery of this ion channel [19].

\section{From nano-scale morphology to near-atomic resolution structure of $I P_{3} R 1$ : how did we get there?}

A number of factors influenced the success of near-atomic structure determination of $\mathrm{IP}_{3} \mathrm{R} 1$ by single particle cryo-EM. In general, single particle cryo-EM analysis requires that the protein of interest is prepared in the form of aqueous solution of monodisperse, identical particles. This requirement poses particular challenges in the purification and stabilization of integral membrane proteins given that their native environment is a lipid bilayer. Key to determining a high-resolution structure of $\mathrm{IP}_{3} \mathrm{R} 1$ was an optimized purification procedure combined with biochemical and functional characterization of the purified channel protein. The cerebellum is the most abundant tissue for $\mathrm{IP}_{3} \mathrm{R} 1$ protein and was used as the primary source for the channel purification $[45,46]$. Choice of the detergent used for the protein solubilization and protein-to-detergent ratio in the protein sample has been proven to be a critical factor in achieving optimal vitrification conditions including the protein stability, ice thickness, particle distribution on an EM grid, that together can affect the quality of cryo$\mathrm{EM}$ data [44]. Another encountered problem for $\mathrm{IP}_{3} \mathrm{R} 1$ is obtaining the protein concentrations sufficient for cryo-specimen preparations. Membrane proteins are particularly 
prone to aggregating during purification and concentration of the detergent solubilized protein sample. In the case of $\mathrm{IP}_{3} \mathrm{R} 1$ studies, a continuous layer of thin carbon support film was used to achieve adhesion and optimal distribution of channel particles on the EM grid. These careful considerations enabled the structure of $\mathrm{IP}_{3} \mathrm{R} 1$ to be solved initially at nanometer [39] and later at near-atomic resolution. However, there are two major factors that allowed us to push the resolution limit in recent cryo-EM studies of $\mathrm{IP}_{3} \mathrm{R} 1$. First, data quality was dramatically improved due to the use of the direct electron detector. This new camera produces substantially higher signal-to-noise ratio at both low and high resolution and allows correcting for beam-induced motion, thereby practically doubling detector resolution compared to previous CCD technology. Second, new powerful image processing algorithms and methods have been designed within the framework of single-particle reconstruction software allowed for sorting out sample heterogeneity due to genuine protein flexibility and conformational variability of $\mathrm{IP}_{3} \mathrm{R} 1$ channel. These technical advances in the cryo-EM field have been extensively discussed in excellent comprehensive reviews [3-5,47].

All together these methodological and technical improvements yielded a $4.7 \AA$ resolution 3D structure of $\mathrm{IP}_{3} \mathrm{R} 1$ based on the gold standard FSC criterion (Fig. 1). The map veracity was further validated with a phase randomization test (Fig. 1d) [48] and by performing independent reconstructions using two different software packages, EMAN2.1 and Relion1.3 (Fig. $1 \mathrm{a}-\mathrm{c}$ ). Notably, the local resolution varies from $3.6 \AA$ to $6.5 \AA$ through the entire 3D reconstruction but was of sufficient quality to trace the backbone topology for 2,327 of 2,750 amino acids and unambiguously identify ten domains that comprise the fulllength $\mathrm{IP}_{3} \mathrm{R} 1$ protein (Fig. 2). Importantly, observed structural features correlate with the resolution assessment excluding a possibility for noise over-fitting during iterative refinement and providing confidence to functional interpretations. TM domain densities were best resolved permitting to clearly define side-chain densities of bulky residues (e.g. F2575, F2586, I2590, D2591, R2597) in the $\mathrm{Ca}^{2+}$ permeation pathway. However, side-chain assignments in the cytoplasmic domains where the density is not sufficiently resolved for rotamer placement were excluded from the final model of $\mathrm{IP}_{3} \mathrm{R} 1$ and not interpreted.

\section{Key domains in $I P_{3} R$ : structure-function conservation}

Recent advent of near atomic resolution structures of $\mathrm{IP}_{3} \mathrm{R} 1$ and RyR1 allows for many parallels to be drawn between the two channels serving to expand our understanding of molecular mechanisms for their activation and gating. $\mathrm{IP}_{3} \mathrm{R}$ and RyR channels largely perform the same intracellular function of $\mathrm{Ca}^{2+}$ transport across the ER/SR membrane, however they respond to different cellular stimuli, and the intensity, shape and duration of $\mathrm{Ca}^{2+}$ signals generated by these channels are defined by their regulators. Each channel function - signal detection, ligand binding, gating - captures specific properties of the protein structure.

Topologically, $\mathrm{Ca}^{2+}$ release channels are members of the tetrameric cation channel superfamily, where each channel monomer contains six transmembrane helices (TM1TM6), of which the last two helices (TM5 and TM6) from four subunits pack in a righthanded bundle forming a single ion-conducting pore in the center of the channel. Helices TM1-TM4 form a peripheral bundle connected to the pore bundle (TM5/TM6) via the 
TM4-5 linker, which structure and position result in swapping of TM domains, i.e TM1TM4 bundle of one subunit interacts with the pore helices from the neighboring subunit. The swapped architecture of TM domains is conserved feature of the tetrameric cation channels. While the overall architecture of the TM domains in $\mathrm{IP}_{3} \mathrm{R} 1$ is similar to that of RyR1 structure, closer inspection reveals important sequence variations (e.g. TM5-6 lumenal and TM2-3 cytosolic loops), which may explain differences in the gating properties of $\mathrm{IP}_{3} \mathrm{R}$ and RyR channels.

The basic structural framework of $\mathrm{IP}_{3} \mathrm{R} 1$ is provided by a unique architecture of the $\mathrm{C}$ terminal helical domains (CTDs, Fig. 2 and 3) that are packed together to form a left-handed bundle around the fourfold channel axis. This bundle is $\sim 80 \AA$ long and spans the entire cytoplasmic region in the tetrameric channel assembly. As a consequence of the structural organization of the CTDs, the N-terminal ligand-binding domains (LBDs: $\beta$-TF1, $\beta$-TF2 and ARM1) connect to the TM domains through a helical linker domain (LNK) lying at the cytosol-membrane interface. This is an unexpected and exciting aspect of $\mathrm{IP}_{3} \mathrm{R} 1$ structure solved by cryo-EM, which was impossible to reconcile with the decade old consensus that signals evoked by $\mathrm{IP}_{3}$ binding to the $\mathrm{N}$-terminus of the channel protein are transmitted to the channel pore formed near the C-terminus via a direct link between the LBD and TM domains [49-51]. In contrast, our structural studies strongly argue against this model and support a mechanism whereby long-range allosteric interactions through the CTD and LNK domains provide the functional coupling between ligand binding and channel opening. On the basis of the $\mathrm{IP}_{3} \mathrm{R} 1$ structure several additional inter- and intra-domain interfaces were identified that might be involved in ligand-induced signal transmission towards the pore. This provides an additional level of channel regulation where multiple regulatory signals merge to control channel gating. This observation is consistent with structural results for RyR [20-25]. However, our analysis reveals that the CTD of RyR1 is structurally homologous to the LNK domain of $\mathrm{IP}_{3} \mathrm{R} 1$ (Fig. 3), suggesting differences in the coupling mechanism underlying allosteric modulation of these two receptors. Notably, the CTDs of tetrameric TRPA1 display the architecture similar to that of $\mathrm{IP}_{3} \mathrm{R} 1$ (Fig. 3a) [17].

An intriguing feature shared by both families of $\mathrm{Ca}^{2+}$ release channels is that the cytoplasmic domains form multiple a-helical solenoid scaffolds (Fig. 3). Given that $\mathrm{Ca}^{2+}$ release channels assemble in vivo as multi-protein complexes with auxiliary regulatory proteins, it is clear that their functionality greatly benefits from the modular solenoid design providing the binding sites for channel binding partners. Sequence analysis indicates that the two channels substantially differ in the number of residues in the full-length protein $\left(\operatorname{IP}_{3} \mathrm{R} 1\right.$ : 2750 amino acids and RyR1: 5037 amino acids) and share only modest sequence homology $(\sim 17 \%)$. However, the alignment of the two structures highlights significant similarity between the two receptors (Fig. 3).

While primary sequence alignments of $\mathrm{IP}_{3} \mathrm{R}$ and RyR families have played a major role in the comparative analyses to tease out channel structure-function, the lack of high-resolution 3D protein structures substantially limited the ability to fully rationalize channel properties. 3D protein folds are generally more conserved than amino acid sequences. Consequently 3D structural comparisons can result in more accurate sequence alignments and thus can be more informative. In the case of $\mathrm{IP}_{3} \mathrm{R}$ and RyR, sequence alignments between such large 
proteins with low overall sequence homology and many stretches of non-homologous regions, are prone to many errors that are dependent on the users choice of algorithm and gap penalties. Recent structural studies of RyR1 clearly demonstrate this point [20,25].

The $\mathrm{Ca}^{2+}$ sensor domains in $\mathrm{IP}_{3} \mathrm{R} 1$ and RyR1 were first described through efforts to associate conserved residues with their functional role. Highly conserved glutamate residues (E4032 in RyR1 and E2101 in $\mathrm{IP}_{3} \mathrm{RI}$ ) were previously identified to play a role in $\mathrm{Ca}^{2+}$ sensitivity for activation of $\mathrm{IP}_{3} \mathrm{RI}$ and RyR1 channels [52,53], and proposed to likely serve an equivalent structure-function role in both channel families. However, both Efremov et al. [20] and des Georges et al. [25] claim that these residues are not structurally equivalent based purely on sequence alignment. In contrast with this, our structural alignment of the ARM3 domain of $\mathrm{IP}_{3} \mathrm{RI}$ and $\mathrm{C}$-solenoid domain of RyR1 has revealed a strongly conserved protein fold (Ca-RMS deviation of $1.8 \AA$ ) with the conserved glutamate residues occupying the same spatial location within their respective domain (Fig. 4). The resulting sequence alignments based on the aligned structures showed additional conserved residues (Fig. 4c), including two residues recently proposed to coordinate $\mathrm{Ca}^{2+}$ for RyR1 (Fig. 4b) [25]. Whether these residues play a role in coordinating $\mathrm{Ca}^{2+}$ in the $\mathrm{IP}_{3} \mathrm{R} 1$ channel is not yet known, and future high-resolution structures of the ligand-bound channel may answer this question.

\section{Conclusions}

$\mathrm{Ca}^{2+}$ release channels were among the first ion channels attempted by single particle cryo-

EM due to their size and relative natural abundance. Remarkable advances in single particle cryo-EM have recently resulted in near-atomic resolution structures of both full-length IP3R1 and RyR1 channels permitting the exploration of their unique gating characteristics [19-24]. A proposed mechanism of ligand-gating based on structural analysis of $I_{3} R$ suggests that structural rearrangements are rather necessary to account for the conductance properties of $\mathrm{IP}_{3} \mathrm{R} 1$ in the open state, however it is not yet obvious how the TM and cytoplasmic domains structurally rearrange upon channel activation and gating. Thus, the current major goal is to solve high-resolution structures of $\mathrm{IP}_{3} \mathrm{R}$ in different functionally relevant states, providing further insights into $\mathrm{IP}_{3} \mathrm{R}$ channel molecular mechanisms.

Until now cryo-EM structural studies of $\mathrm{IP}_{3} \mathrm{R} 1$ have been conducted on the tetrameric $\mathrm{IP}_{3} \mathrm{R} 1$ purified from native source, yet the regions for which the $\mathrm{IP}_{3} \mathrm{R} 1$ backbone structure has yet to be resolved overlap with the known locations for alternative splicing and regions of posttranslational modifications. This level of heterogeneity has complicated structure-functional studies of $\mathrm{IP}_{3} \mathrm{R}$. Future structural studies will greatly benefit from the production of recombinant $I_{3} R$ proteins, which are expected to be more homogeneous and advantageous for achieving higher resolution structures by single-particle cryo-EM. Additionally, the nature of lipid and solubilizing detergent affects the stability of the protein and likelihood of high-resolution structure determination by cryo-EM [54,55]. It has been demonstrated that purified membrane protein nano-disc preparations are particularly stable and allow the protein to be maintained at high concentrations suitable for cryo-EM studies $[8,12]$. However, one must keep in mind that this approach is prone to a general limitation of singleparticle cryo-EM when exploring structure-function of ion channels: both faces of the 
channel assembly are exposed to the same environment, while in vivo ion channels function in the biological membrane and are exposed to an essentially asymmetric cellular milieu. From this perspective, structural studies of ion channels reconstituted into lipid vesicles will likely have a high impact on structural analysis as this approach reflects both the presence of lipids, which can effect activity, and also allows to explore the structural basis of unique gating characteristics in a native-like environment [56,57].

Furthermore, based on numerous functional studies multiple conformations with different lifetimes and stability likely underlie $\mathrm{IP}_{3} \mathrm{R}$ 's functional states. Approaches to reduce conformational flexibility of $\mathrm{IP}_{3} \mathrm{R}$ include stabilization of the channel conformation by binding of specific ligands, which would promote formation of stable channel complexes suitable for high-resolution structure determination. Overall, the field is now taking advantage of the high-resolution and high-throughput single particle cryo-EM, which together with advanced biochemical and physiological studies, will lead to increased rate of structure-function discoveries in the near future.

\section{Acknowledgments}

The authors thank Steve Ludtke for providing 3D reconstruction of $\mathrm{IP}_{3} \mathrm{RI}$ generated with EMAN2.1, and the National Institutes of Health (R01 GM072804), the American Heart Association (16GRNT29720001) and the Muscular Dystrophy Association (295138) for providing support.

\section{References and recommended reading}

Papers of particular interest, published within the period of review, have been highlighted as

- of special interest

•• of outstanding interest

1. Durham WJ, Wehrens XH, Sood S, Hamilton SL. Diseases associated with altered ryanodine receptor activity. Subcell Biochem. 2007; 45:273-321. [PubMed: 18193641]

2. Bezprozvanny, I. Inositol 1,4,5-tripshosphate receptor, calcium signalling and Huntington's disease. Harris, JR., editor. Vol. 45. Springer; 2007.

3. Milne JL, Borgnia MJ, Bartesaghi A, Tran EE, Earl LA, Schauder DM, Lengyel J, Pierson J, Patwardhan A, Subramaniam S. Cryo-electron microscopy-a primer for the non-microscopist. FEBS J. 2013; 280:28-45. http://dx.doi.org/10.1111/febs.12078. [PubMed: 23181775]

4. Kuhlbrandt W. Cryo-EM enters a new era. Elife. 2014; 3:e03678. http://dx.doi.org/10.7554/eLife. 03678. [PubMed: 25122623]

5. Subramaniam S, Earl LA, Falconieri V, Milne JL, Egelman EH. Resolution advances in cryo-EM enable application to drug discovery. Curr Opin Struct Biol. 2016; 41:194-202. http://dx.doi.org/ 10.1016/j.sbi.2016.07.009. [PubMed: 27552081]

6••. Bartesaghi A, Merk A, Banerjee S, Matthies D, Wu X, Milne JLS, Subramaniam S. 2.2 A resolution cryo-EM structure of $\beta$-galactosidase in complex with a cell-permeant inhibitor. Science. 2015; 348:1147-1151. http://dx.doi.org/10.1126/science.aab1576 This paper represents a breakthrough in the cryo-EM field whereby the structure of the $465 \mathrm{kDa} \beta$-galactosidase protein was solved to atomic resolution permitting the visulaization of features typically observed in structures determined by x-ray crystallography solved at the same resolution, including amino acid side-chain densities, carbonyl moieties, coordinated $\mathrm{Mg} 2+$ and strongly bound water molecules. [PubMed: 25953817]

7••. Merk A, Bartesaghi A, Banerjee S, Falconieri V, Rao P, Davis MI, Pragani R, Boxer MB, Earl LA, Milne JL, et al. Breaking Cryo-EM Resolution Barriers to Facilitate Drug Discovery. Cell. 2016; 
165:1698-1707. http://dx.doi.org/10.1016/j.cell.2016.05.040. The paper reports near-atomic resoltuion structures of two small soluble enzymes (the $144 \mathrm{kDa}$ lactate dehydrogenase and the $93 \mathrm{kDa}$ isocytrate dehydrogenase) determined by single particle cryo-EM. The study demonstrates the power of cryo-EM technology, breaking resoltuion and size limits for structure determination of proteins and becoming a central tool in structural biology. [PubMed: 27238019]

8••. Gao Y, Cao E, Julius D, Cheng Y. TRPV1 structures in nanodiscs reveal mechanisms of ligand and lipid action. Nature. 2016; 534:347-351. http://dx.doi.org/10.1038/nature17964. This paper utlizes the combined approach of cryo-EM and lipid nanodisc technology to determine the structure of the solubilized TRPV1 ion channel in a more native-like environment. This progressive approach revealed details of phospholipid and ligand regulation of the channel. [PubMed: 27281200]

9. Jiang J, Pentelute BL, Collier RJ, Zhou ZH. Atomic structure of anthrax protective antigen pore elucidates toxin translocation. Nature. 2015; 521:545-549. http://dx.doi.org/10.1038/nature14247. [PubMed: 25778700]

10••. Bai XC, Yan C, Yang G, Lu P, Ma D, Sun L, Zhou R, Scheres SH, Shi Y. An atomic structure of human gamma-secretase. Nature. 2015; 525:212-217. http://dx.doi.org/10.1038/nature14892. The paper reports the $3.7 \AA$-resolution strcutrue of gamma-secretase, an integral membrane protein with a molecualr weight of $\sim 170 \mathrm{kDa}$. This study demonstrates that rapidly developing cryo-EM technologies are applicable to structure determination of relatively small membrane proteins. [PubMed: 26280335]

11••. Liao M, Cao E, Julius D, Cheng Y. Structure of the TRPV1 ion channel determined by electron cryo-microscopy. Nature. 2013; 504:107-112. http://dx.doi.org/10.1038/nature12822. This seminal paper reports the first structure of an ion channel determined by single particle cryo-EM to near-atomic resoltuion. This is the first demonstration of the emerging power and advantage of cryo-EM, in which a high-resolution structure of a membrane protein can be achieved without crystallization. [PubMed: 24305160]

12••. Shen PS, Yang X, DeCaen PG, Liu X, Bulkley D, Clapham DE, Cao E. The Structure of the Polycystic Kidney Disease Channel PKD2 in Lipid Nanodiscs. Cell. 2016; 167:763-773. e711. doi: http://dx.doi.org/10.1016/j.cell.2016.09.048. The paper reports the first structure of PKD2 ion channel in lipid nanodics at $3.0 \AA$ resolution. It illustrates the powerful applicability of nanodiscs technology in combination with single-particle cryo-EM to determine high-resolution structures on membrane proteins. [PubMed: 27768895]

13. Wu J, Yan Z, Li Z, Qian X, Lu S, Dong M, Zhou Q, Yan N. Structure of the voltage-gated calcium channel Cavl. 1 at $3.6 \AA$ resolution Nature. 2016; 537:191-196. http://dx.doi.org/10.1038/ nature 19321.

14. Whicher JR, MacKinnon R. Structure of the voltage-gated $\mathrm{K}^{+}$channel Eagl reveals an alternative voltage sensing mechanism. Science. 2016; 353:664-669. http://dx.doi.org/10.1126/ science.aaf8070. [PubMed: 27516594]

15. Zubcevic L, Herzik MA Jr, Chung BC, Liu Z, Lander GC, Lee SY. Cryo-electron microscopy structure of the TRPV2 ion channel. Nat Struct Mol Biol. 2016; 23:180-186. http://dx.doi.org/ 10.1038/nsmb.3159. [PubMed: 26779611]

16. Matthies D, Dalmas O, Borgnia MJ, Dominik PK, Merk A, Rao P, Reddy BG, Islam S, Bartesaghi A, Perozo E, et al. Cryo-EM Structures of the Magnesium Channel CorA Reveal Symmetry Break upon Gating. Cell. 2016; 164:747-756. http://dx.doi.org/10.1016/j.cell.2015.12.055. [PubMed: 26871634]

17. Paulsen CE, Armache JP, Gao Y, Cheng Y, Julius D. Structure of the TRPA1 ion channel suggests regulatory mechanisms. Nature. 2015; 520:511-517. http://dx.doi.org/10.1038/nature14367. The paper reports the near-atomic resolution cryo-EM structure of TRPA1 revealing a unique ahelical arranegment of the C-terminal domains in the tetrameric channel assembly. [PubMed: 25855297]

18. Hite RK, Yuan P, Li Z, Hsuing Y, Walz T, MacKinnon R. Cryo-electron microscopy structure of the Slo2.2 $\mathrm{Na}^{+}$-activated $\mathrm{K}^{+}$channel. Nature. 2015; 527:198-203. http://dx.doi.org/10.1038/ nature14958. [PubMed: 26436452]

19••. Fan G, Baker ML, Wang Z, Baker MR, Sinyagovskiy PA, Chiu W, Ludtke SJ, Serysheva II. Gating machinery of InsP3R channels revealed by electron cryomicroscopy. Nature. 2015; 
527:336-341. http://dx.doi.org/10.1038/nature15249. The paper reports the first near-atomic resolution of structure of the full-legth tetrameric IP3R1 channel in the apo-state determined by single particle cryo-EM. The study provides critical insights into the $3 \mathrm{D}$ architecture and structural coupling underlying ligand-induced channel gating. [PubMed: 26458101]

20•. Efremov RG, Leitner A, Aebersold R, Raunser S. Architecture and conformational switch mechanism of the ryanodine receptor. Nature. 2015; 517:39-43. http://dx.doi.org/10.1038/ nature13916. The paper reports cryo-EM structures of RyR1 in apo- and ligand-bound states detremined by single particle cryo-EM. [PubMed: 25470059]

21 . Zalk R, Clarke OB, des Georges A, Grassucci RA, Reiken S, Mancia F, Hendrickson WA, Frank J, Marks AR. Structure of a mammalian ryanodine receptor. Nature. 2015; 517:44-49. http:// dx.doi.org/10.1038/nature13950. The paper reports a near-atomic resolution structure of the fulllength tetrameric RyR1 determined by single particle cryo-EM. [PubMed: 25470061]

22•. Yan Z, Bai XC, Yan C, Wu J, Li Z, Xie T, Peng W, Yin CC, Li X, Scheres SH, et al. Structure of the rabbit ryanodine receptor RyR1 at near-atomic resolution. Nature. 2015; 517:50-55. http:// dx.doi.org/10.1038/nature14063. The paper reports a near-atomic resolution structure of the fulllength tetrameric RyR1 determined by single particle cryo-EM. [PubMed: 25517095]

23. Wei R, Wang X, Zhang Y, Mukherjee S, Zhang L, Chen Q, Huang X, Jing S, Liu C, Li S, et al. Structural insights into $\mathrm{Ca}^{2+}$-activated long-range allosteric channel gating of RyR1. Cell Res. 2016; 26:977-994. http://dx.doi.org/10.1038/cr.2016.99. [PubMed: 27573175]

24. Bai XC, Yan Z, Wu J, Li Z, Yan N. The Central domain of RyR1 is the transducer for long-range allosteric gating of channel opening. Cell Res. 2016; 26:995-1006. http://dx.doi.org/10.1038/cr. 2016.89. [PubMed: 27468892]

25••. des Georges A, Clarke OB, Zalk R, Yuan Q, Condon KJ, Grassucci RA, Hendrickson WA, Marks AR, Frank J. Structural Basis for Gating and Activation of RyR1. Cell. 2016; 167:145-157. e117. doi: http://dx.doi.org/10.1016/j.cell.2016.08.075. This paper presents several cryo-EM reconstructions of RyR1 in multiple functional states revealing the structural basis of channel gating and ligand-dependent activation. [PubMed: 27662087]

26. Foskett JK, White C, Cheung KH, Mak DO. Inositol trisphosphate receptor $\mathrm{Ca}^{2+}$ release channels. Physiol Rev. 2007; 87:593-658. [PubMed: 17429043]

27. Taylor CW, Tovey SC. $\mathrm{IP}_{3}$ receptors: toward understanding their activation. Cold Spring Harb Perspect Biol. 2010; 2:a004010. http://dx.doi.org/10.1101/cshperspect.a004010. [PubMed: 20980441]

28. Bosanac I, Alattia JR, Mal TK, Chan J, Talarico S, Tong FK, Tong KI, Yoshikawa F, Furuichi T, Iwai M, et al. Structure of the inositol 1,4,5-trisphosphate receptor binding core in complex with its ligand. Nature. 2002; 420:696-700. [PubMed: 12442173]

29. Bosanac I, Yamazaki H, Matsu-Ura T, Michikawa T, Mikoshiba K, Ikura M. Crystal structure of the ligand binding suppressor domain of type 1 inositol 1,4,5-trisphosphate receptor. Mol Cell. 2005; 17:193-203. [PubMed: 15664189]

30. Lin CC, Baek K, Lu Z. Apo and $\mathrm{InsP}_{3}$-bound crystal structures of the ligand-binding domain of an $\mathrm{InsP}_{3}$ receptor. Nat Struct Mol Biol. 2011; 18:1172-1174. [PubMed: 21892169]

31. Seo MD, Velamakanni S, Ishiyama N, Stathopulos PB, Rossi AM, Khan SA, Dale P, Li C, Ames JB, Ikura M, et al. Structural and functional conservation of key domains in $\mathrm{InsP}_{3}$ and ryanodine receptors. Nature. 2012; 483:108-112. http://dx.doi.org/10.1038/nature10751. [PubMed: 22286060]

32. Chan J, Whitten AE, Jeffries CM, Bosanac I, Mal TK, Ito J, Porumb H, Michikawa T, Mikoshiba $\mathrm{K}$, Trewhella J, et al. Ligand-induced conformational changes via flexible linkers in the aminoterminal region of the inositol 1,4,5-trisphosphate receptor. J Mol Biol. 2007; 373:1269-1280. [PubMed: 17915250]

33. Li C, Enomoto M, Rossi AM, Seo MD, Rahman T, Stathopulos PB, Taylor CW, Ikura M, Ames JB. CaBP1, a neuronal $\mathrm{Ca}^{2+}$ sensor protein, inhibits inositol trisphosphate receptors by clamping intersubunit interactions. Proc Natl Acad Sci U S A. 2013; 110:8507-8512. http://dx.doi.org/ 10.1073/pnas. 1220847110. [PubMed: 23650371]

34. da Fonseca PC, Morris SA, Nerou EP, Taylor CW, Morris EP. Domain organization of the type 1 inositol 1,4,5-trisphosphate receptor as revealed by single-particle analysis. Proc Natl Acad Sci U S A. 2003; 100:3936-3941. [PubMed: 12651956] 
35. Hamada K, Terauchi A, Mikoshiba K. Three-dimensional rearrangements within inositol 1, 4, 5trisphosphate receptor by calcium. J Biol Chem. 2003; 278:52881-52889. [PubMed: 14593123]

36. Jiang QX, Thrower EC, Chester DW, Ehrlich BE, Sigworth FJ. Three-dimensional structure of the type 1 inositol 1,4,5-trisphosphate receptor at $24 \AA$ resolution. Embo J. 2002; 21:3575-3581. [PubMed: 12110570]

37. Sato C, Hamada K, Ogura T, Miyazawa A, Iwasaki K, Hiroaki Y, Tani K, Terauchi A, Fujiyoshi Y, Mikoshiba K. Inositol 1,4,5-trisphosphate receptor contains multiple cavities and L-shaped ligandbinding domains. J Mol Biol. 2004; 336:155-164. [PubMed: 14741211]

38. Serysheva II, Bare DJ, Ludtke SJ, Kettlun CS, Chiu W, Mignery GA. Structure of the type 1 inositol 1,4,5-trisphosphate receptor revealed by electron cryomicroscopy. J Biol Chem. 2003; 278:21319-21322. [PubMed: 12714606]

39•. Ludtke SJ, Tran TP, Ngo QT, Moiseenkova-Bell VY, Chiu W, Serysheva II. Flexible architecture of $\mathrm{IP}_{3} \mathrm{R} 1$ by Cryo-EM. Structure. 2011; 19:1192-1199. http://dx.doi.org/10.1016/j.str. 2011.05.003. The paper reports the first 3D structure of IP3R1 unambiguosly determined at nanometer resolution by single particle cryo-EM. [PubMed: 21827954]

40••. Murray SC, Flanagan J, Popova OB, Chiu W, Ludtke SJ, Serysheva II. Validation of cryo-EM structure of $\mathrm{IP}_{3} \mathrm{R} 1$ channel. Structure. 2013; 21:900-909. http://dx.doi.org/10.1016/j.str. 2013.04.016. The paper reports the validation of 3D structure of IP3R1 by the use of several approaches: independent resonstructions with five different softwares (EMAN1, EMAN2, Relion, Imagic, SPARX), tilt-pair analysis, class-average/map comparisons, and gold standard FSC criterion. [PubMed: 23707684]

41. Rosenthal PB, Henderson R. Optimal determination of particle orientation, absolute hand, and contrast loss in single-particle electron cryomicroscopy. J Mol Biol. 2003; 333:721-745. The paper describes the tilt-pair analysis that tests the valididly of a 3D reconstrcution at lowresoltuion and demonstrates the accuracy of orientation determination. [PubMed: 14568533]

42. Henderson R, Chen S, Chen JZ, Grigorieff N, Passmore LA, Ciccarelli L, Rubinstein JL, Crowther RA, Stewart PL, Rosenthal PB. Tilt-pair analysis of images from a range of different specimens in single-particle electron cryomicroscopy. J Mol Biol. 2011; 413:1028-1046. http://dx.doi.org/ 10.1016/j.jmb.2011.09.008. [PubMed: 21939668]

43. Scheres SH, Chen S. Prevention of overfitting in cryo-EM structure determination. Nat Methods. 2012; 9:853-854. http://dx.doi.org/10.1038/nmeth.2115. The paper reports that estimation of resolution using frequency limited refinement of independent half-maps (“gold standard FSC") reduces overfitting of noise but does not adversely affect the final map resolution, therefore providing a realistic resolution estimate. [PubMed: 22842542]

44. Ludtke SJ, Serysheva II. Single-particle cryo-EM of calcium release channels: structural validation. Curr Opin Struct Biol. 2013; 23:755-762. http://dx.doi.org/10.10167j.sbi.2013.06.003. [PubMed: 23831288]

45. Sudhof TC, Newton CL, Archer BT 3rd, Ushkaryov YA, Mignery GA. Structure of a novel InsP 3 receptor. Embo J. 1991; 10:3199-3206. [PubMed: 1655411]

46. Wojcikiewicz RJ. Type I, II, and III inositol 1,4,5-trisphosphate receptors are unequally susceptible to down-regulation and are expressed in markedly different proportions in different cell types. J Biol Chem. 1995; 270:11678-11683. [PubMed: 7744807]

47. Liao M, Cao E, Julius D, Cheng Y. Single particle electron cryo-microscopy of a mammalian ion channel. Curr Opin Struct Biol. 2014; 27:1-7. http://dx.doi.org/10.1016Zj.sbi.2014.02.005. [PubMed: 24681231]

48• Chen S, McMullan G, Faruqi AR, Murshudov GN, Short JM, Scheres SH, Henderson R. Highresolution noise substitution to measure overfitting and validate resolution in $3 \mathrm{D}$ structure determination by single particle electron cryomicroscopy. Ultramicroscopy. 2013; 135:24-35. http://dx.doi.org/10.1016/j.ultramic.2013.06.004. The paper describes a test for detection of overfitting by making use of images where the phases at high-resolution spatial ferquences are randomized or completely subsituted by noise. The test allows for measurement of the amount of signal and the amount of noise that is present in the final 3D reconstruciton. [PubMed: 23872039] 
49. Boehning D, Joseph SK. Direct association of ligand-binding and pore domains in homo- and heterotetrameric inositol 1,4,5-trisphosphate receptors. Embo J. 2000; 19:5450-5459. [PubMed: 11032812]

50. Yoshikawa F, Iwasaki H, Michikawa T, Furuichi T, Mikoshiba K. Trypsinized cerebellar inositol 1,4,5-trisphosphate receptor. Structural and functional coupling of cleaved ligand binding and channel domains. J Biol Chem. 1999; 274:316-327. [PubMed: 9867846]

51. Schug ZT, Joseph SK. The role of the S4-S5 linker and C-terminal tail in inositol 1,4,5trisphosphate receptor function. J Biol Chem. 2006; 281:24431-24440. [PubMed: 16815846]

52. Du GG, MacLennan DH. Functional consequences of mutations of conserved, polar amino acids in transmembrane sequences of the $\mathrm{Ca}^{2+}$ release channel (ryanodine receptor) of rabbit skeletal muscle sarcoplasmic reticulum. J Biol Chem. 1998; 273:31867-31872. [PubMed: 9822655]

53. Miyakawa T, Mizushima A, Hirose K, Yamazawa T, Bezprozvanny I, Kurosaki T, Iino M. Ca(2+)sensor region of IP(3) receptor controls intracellular Ca(2+) signaling. Embo J. 2001; 20:16741680. [PubMed: 11285231]

54. Baker MR, Fan G, Serysheva II. Single-Particle Cryo-EM of the Ryanodine Receptor Channel in an Aqueous Environment. Eur J Transl Myol. 2015; 25:4803. http://dx.doi.org/10.4081/ejtm. 2015.4803. [PubMed: 26913144]

55. Serysheva II. Toward a high-resolution structure of IP(3)R channel. Cell Calcium. 2014; 56:125132. http://dx.doi.org/10.1016/j.ceca.2014.08.002. [PubMed: 25159857]

56•. Wang L, Sigworth FJ. Structure of the BK potassium channel in a lipid membrane from electron cryomicroscopy. Nature. 2009; 461:292-295. This paper reports the first cryo-EM structure of the voltage-activated $\mathrm{K}+$ channel $(\mathrm{BK})$ reconstituted into small unilamellar vesicle. The paper describes a new random spherically constrained single-particle recosntruction technique. [PubMed: 19718020]

57. Wang L, Sigworth FJ. Liposomes on a streptavidin crystal: a system to study membrane proteins by cryo-EM. Methods Enzymol. 2010; 481:147-164. http://dx.doi.org/10.1016/ S0076-6879(10)81007-9. [PubMed: 20887857] 


\section{Highlights}

- $\quad$ Full-length tetrameric structure of $\mathrm{IP}_{3} \mathrm{R} 1$ has been solved at $4.7 \AA$ resolution.

- The overall structure was validated by multiple methods.

- The central four-helix bundle provides a supporting scaffold for the cytosolic assembly.

- $\quad$ Direct structural coupling between the $\mathrm{C}$-terminal and $\mathrm{IP}_{3}$-binding domains is revealed.

- Versatility of $\mathrm{Ca}^{2+}$ signaling via $\mathrm{IP}_{3} \mathrm{R}$ is founded in the architecture of cytosolic domains. 
(a)

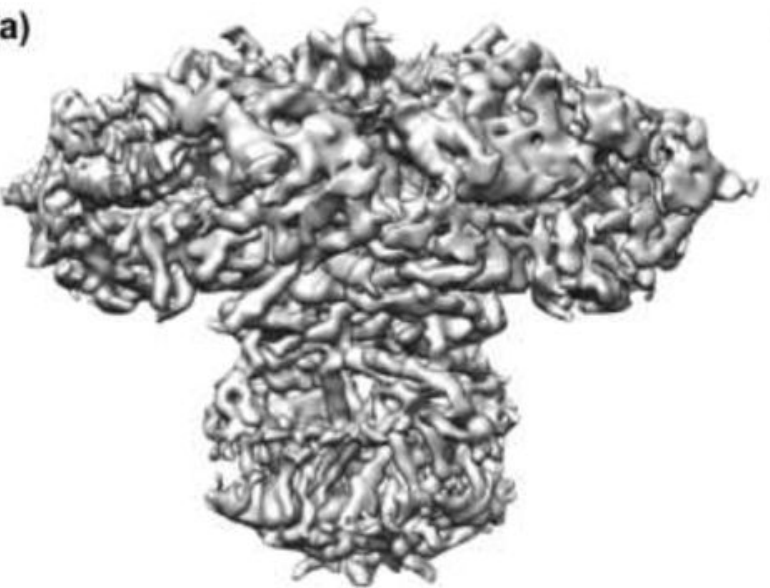

(c)

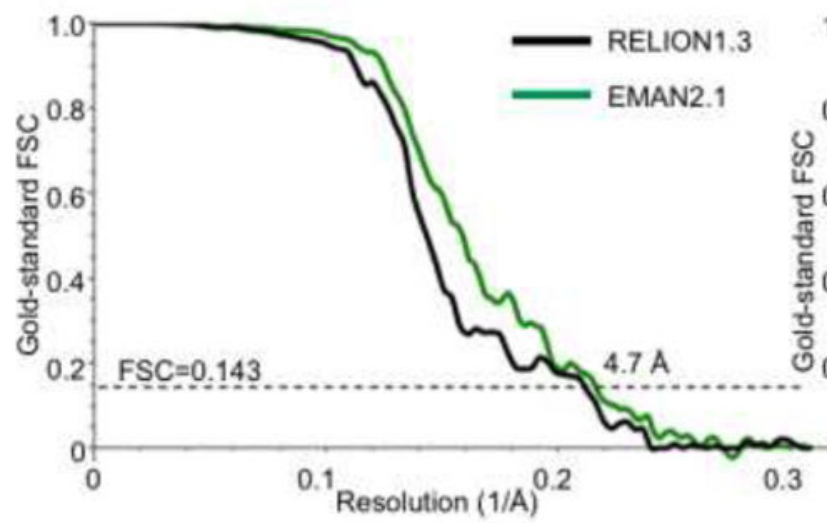

(b)

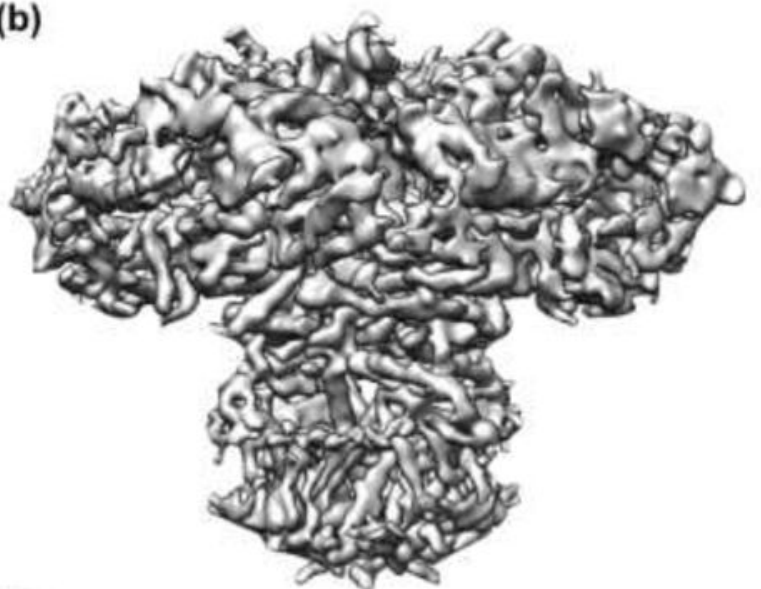

(d)

Figure 1. Resolution assessment and structure validation

Two 3D reconstructions of $\mathrm{IP}_{3} \mathrm{R} 1$ were performed entirely independently with Relion 1.3 (a) and EMAN 2.1 (b) software packages using the same cryo-EM data. (c) Shown are the goldstandard FSC curves [41,43] for the final 3D reconstructions generated with Relion1.3 (black line) and EMAN2.1 (green line). (d) The FSC profiles for the Relion1.3 reconstructions: the blue line shows the FSC curve computed for the data in which the phases were randomized from $6 \AA$ to Nyquist; the gold standard FSC curve is shown with a black line and is nearly identical to the "true FSC" (red line) calculated as described in [48]. 
(a)

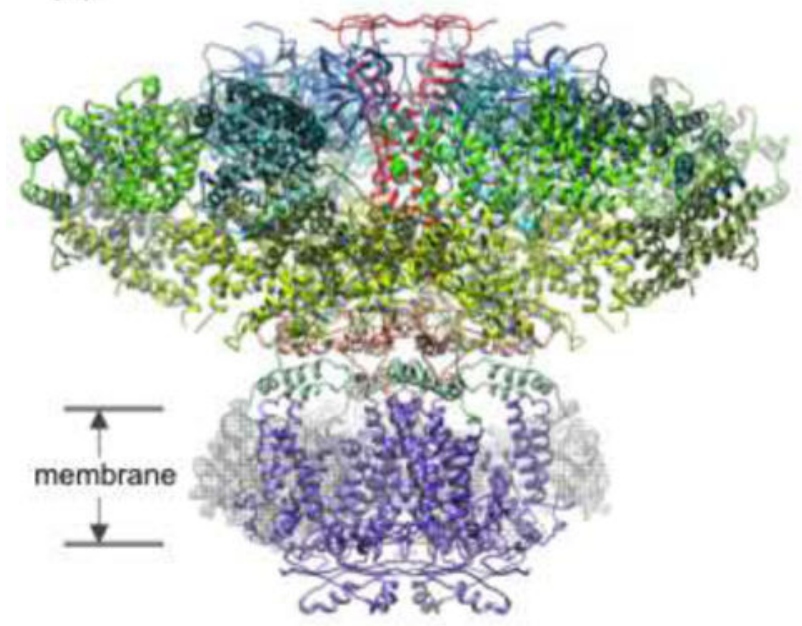

(c)
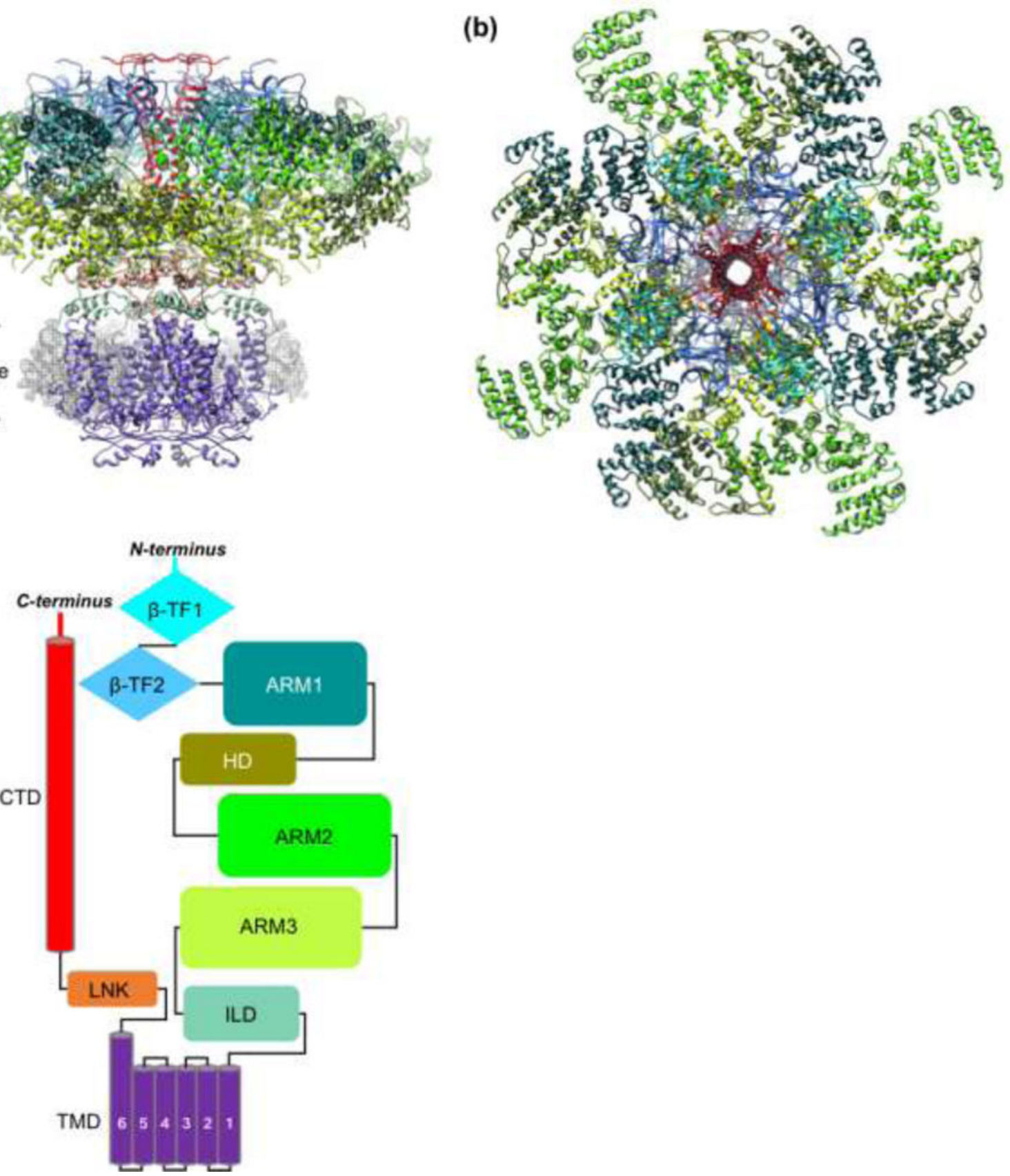

Figure 2. Structure of rat cerebellar $I_{P_{3}} R 1$ determined by single-particle cryo-EM

Orthogonal views for the $\mathrm{IP}_{3} \mathrm{R} 1$ channel: (a) view along the membrane plane; the detergentphospholipid belt is observed at low density threshold (semi-transparent gray mesh) and denotes the position of the channel complex in the membrane with the cytosolic domains facing up; (b) view down the four-fold axis from the cytosolic side. (c) Topological scheme of the ten domains identified in the individual $\mathrm{IP}_{3} \mathrm{R} 1$ subunit. 
(a)
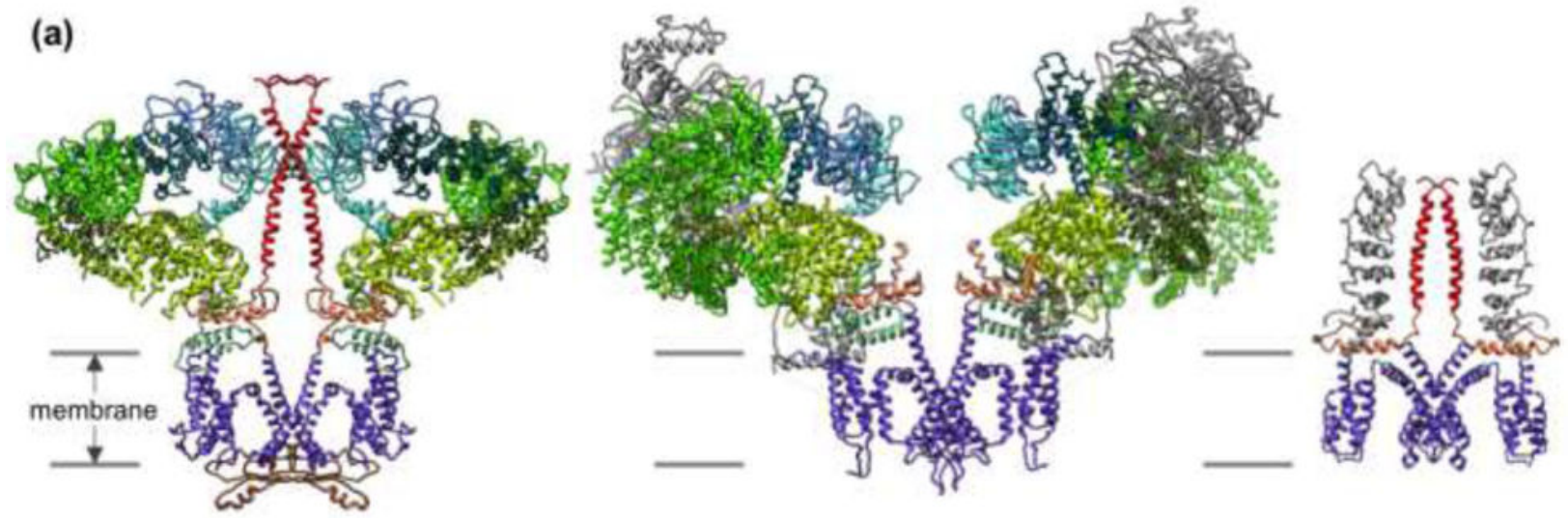

(b)

\begin{tabular}{|c|c|c|c|c|c|c|c|c|c|}
\hline $\begin{array}{c}\text { RyR1 } \\
\text { domains }\end{array}$ & NTD-A & NTO-B & Nsol & SPRY1 & Repeats 182 & & SPRY3 & hol & Bsol \\
\hline Residues & $1-208$ & 209-392 & $393-627$ & $628-849$ & $850-1054$ & $1055-1241$ & $1242-1656$ & 1657.2144 & $2145-3613$ \\
\hline $\begin{array}{c}\text { IP3R1 } \\
\text { domains }\end{array}$ & B-TF1 & A-TF2 & ARM1 & - & - & - & - & HD & ARM2 \\
\hline Residues & $1-225$ & $226-435$ & $436-714$ & - & - & - & - & $725-1079$ & $1030-1494$ \\
\hline Ca-rmsd (A) & 1.7 & 1.8 & 2.5 & - & - & - & - & 2.5 & 3 \\
\hline $\begin{array}{l}\text { " pairs } \\
\text { aligned }\end{array}$ & 135 & 117 & 189 & - & - & - & - & 107 & 208 \\
\hline
\end{tabular}

\begin{tabular}{|c|c|c|c|c|c|c|c|c|c|c|}
\hline $\begin{array}{l}\text { Aypla } \\
\text { domains }\end{array}$ & $\begin{array}{c}\text { Repeats } \\
384\end{array}$ & & CSol & & Taf & pVSD & & Pore & & - \\
\hline Residues & $2735-2938$ & $3614-3666$ & $3667-4174$ & $4060-4134$ & 4175.4253 & $4541-4819$ & $4666-4786$ & $4820-4956$ & 4957.5037 & - \\
\hline $\begin{array}{l}\text { IP3R1 } \\
\text { domains }\end{array}$ & - & - & ARMB & - & ILD & TM 1-TM4 & - & $\begin{array}{l}\text { TMA-5:- } \\
\text { TMMS }\end{array}$ & tWK & CTD \\
\hline Residues & - & - & $1594-2192$ & - & $2193-2272$ & $2273-2427$ & - & $2428-2601$ & $2602-2681$ & $2682-2750$ \\
\hline Ca-rmsd $(A)$ & - & - & 28 & - & 23 & & 1.7 & & 2.1 & - \\
\hline $\begin{array}{l}\text { "pairs } \\
\text { aligned }\end{array}$ & - & - & 223 & - & 57 & & 134 & & 68 & - \\
\hline
\end{tabular}

Figure 3. Structural conservation of ion channels. (a)

Structures of IP 3 R1 (3jav), RyR1 (5tb0) and TRPA1 (3j9p) viewed along the membrane plane. (b) Correspondence of domains between $\mathrm{IP}_{3} \mathrm{R} 1$ and RyR1 based on their structural alignments. Reported are Ca RMSDs for the aligned residue pairs. Structure comparison was performed on the entire domains with the following exceptions: ARM1 is aligned with NSol domains and partial JSol domain (residues 1657-1720), the remaining portion of the JSol domain (residues 1721-2144) is aligned with HD, and BSol residues 2145-2599 are aligned with residues 1119-1494 from ARM2. Structurally conserved domains are colorcoded based on $\mathrm{IP}_{3} \mathrm{R} 1$. 
(a)

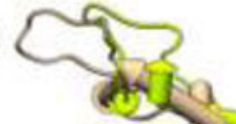
कि?

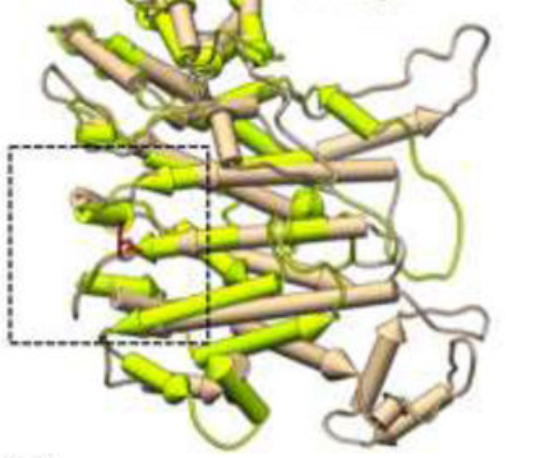

(b)

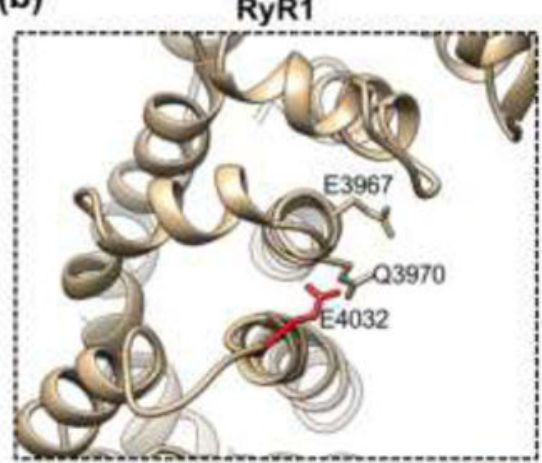

(c)

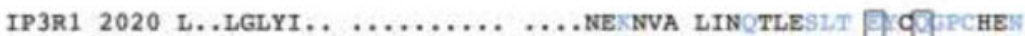
RYR1 3931 SDFY....WY YSGKDVIEEQ GKRNFSIKANS VAKOVFNSLT E IDGPCTGI IP3R1 2052 QNCIATHES. NGIDIITALI LN.D..I.. NP.... LGK KRNDEVLELK RYR1 3977 CQSLAHSRLW DAVVG...F. L.HVFAHMMM KLAQDSSQIE LLKEILDLQ.

IP3R1 2090 NNASKLLIAI MESRH..DSE NAERILYNM. .RPK..ELVE . .VIK.KAY. RYR1 4021 KDMVVMLCSL L GNVVNGMI AROMVDNLVE SS..SWV..E MIL.. KFFDM
IP $\mathbf{P}_{3} \mathbf{1}$
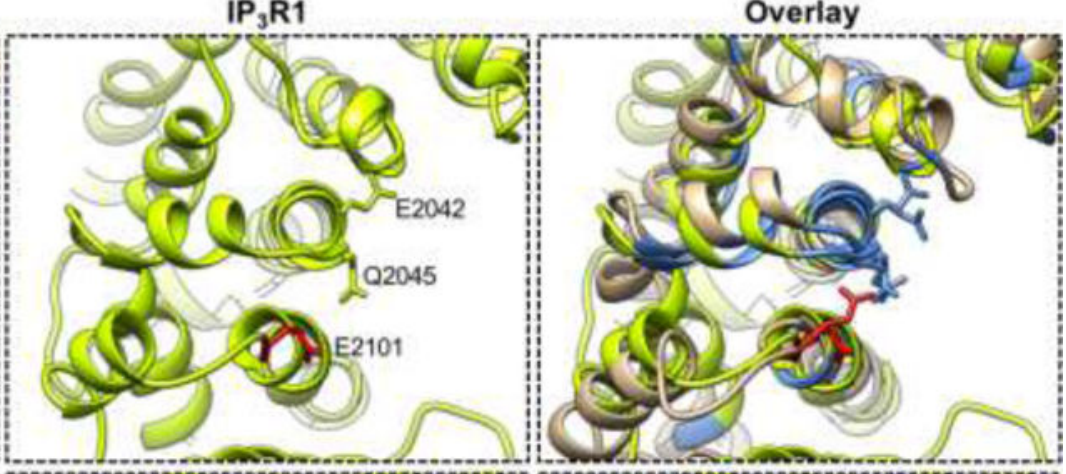

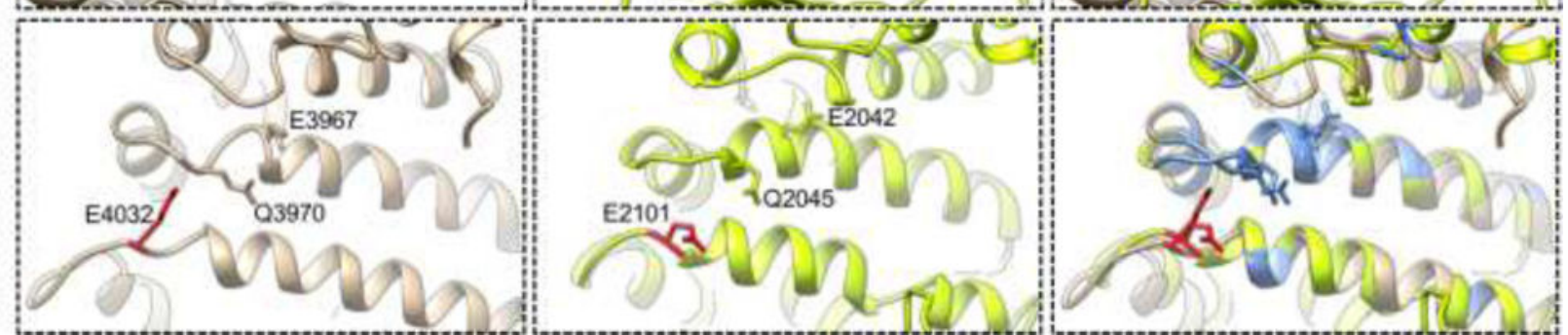

Figure 4. Structural conservation of putative $\mathrm{Ca}^{2+}$ sensor region. (a)

The armadillo repeat domain 3 (ARM3) of $\mathrm{IP}_{3} \mathrm{R} 1$ (3jav) and the C-solenoid of RyR1 (5tb0) share structural and sequence conservation. $\mathrm{IP}_{3} \mathrm{R} 1$ is colored chartreuse and RyR1 is colored tan. Ca RMSD is $1.8 \AA$. Dashed line indicates $\mathrm{Ca}^{2+}$ binding pocket identified in RyR1 [25]. The conserved residues E2101 ( $\left.\mathrm{IP}_{3} \mathrm{R} 1\right)$ and E4032 (RyR1) that have been implicated in channel $\mathrm{Ca}^{2+}$ sensitivity are colored red $(\mathbf{a}-\mathbf{c})$. (b) Two zoomed-in views of the RyR1 $\mathrm{Ca}^{2+}$ binding pocket (left panels) and the structurally equivalent residues of $\mathrm{IP}_{3} \mathrm{R} 1$ (middle panels) are shown. Right panels show the overlay of RyR1 and $\mathrm{IP}_{3} \mathrm{R} 1$ putative $\mathrm{Ca}^{2+}$ binding pockets with all identical residues colored blue. (c) Sequence alignment based on the structural overlap for the ARM3 and C-solenoid domains is shown. Identical residues are colored blue and structurally equivalent residues in $\mathrm{IP}_{3} \mathrm{R} 1$ based on the putatively identified RyR1 $\mathrm{Ca}^{2+}$ binding pocket are indicated with a box. 